\title{
Design and Realization of Power Plant Multi-model Simulation System Based on the EDPF-NT Plus System
}

\author{
Xu Peng, Dong Ze \\ Hebei Engineering Research Center of Simulation \& Optimized Control for Power Generation \\ North China Electric Power University \\ Baoding, China \\ feihua110@sina.com
}

\begin{abstract}
According to the diversified needs of modern power plant simulator training, and as far as possible to reduce the equipment cost, the design of power plant multi-model simulation system ( STSManageCenter) based on the EDPF-NT plus system fully meets the requirements of the scene. This paper introduced the hardware and software configuration in detail, effectively combined the support system of simulation (STS). With a simple graphical interface, realizing the control of synthesized system including Support System, Communication System and DCS. Thus it eased the difficulty level greatly, and realized the function of multi-model in the existing platform.
\end{abstract}

Keywords-component; EDPF-NT+; multi-model simulation; power plant simulation; human-robot interaction

\section{INTRODUCTION}

Simulation technology was adopted in analog computer when the early stage of study in our country. And it turns into digital simulation later [1]. The technology of power plant developed rapidly with the continued improvement of demand for domestic power production. Its important position apparent gradually, because of its effectiveness, economic security, and repeatability. In order to ensure the safety of production process, a lot of simulators are put into operation. It makes a significant contribution to the power industry for the high efficiency and safety in production.

On the platform of Simulation training system, the real time mathematical model can be setup and operation by simulating the field production process and accident [2], etc. Thus the environment of training for operators can be realized. The simulation support system is the core of the support of simulation software, and it is responsible to software's development, commissioning, operation and management. What's more, the simulator directly depends on its quality and level. However, only the simple task can operate makes the shortage of the training, and the less efficient as a result. Therefore a multi-model system STSManageCenter's design becomes the key to solve the problem.

\section{DESIGN OF DCS}

DCS simulation system includes man-machine interface and control logic, and its data collecting sites usually have several thousand to tens of its data. DCS of Guodian Zhishen can meet high parameters, large capacity. It reflects very good performance in the subcritical, supercritical and ultra-supercritical unit control.

EDPF-NT+, which is the distributed control system, developed based on embedded computer system with the latest technology and PROFIBUS technology. Each I/O module has its independent PROFIBUS communication nodes, with the multiple characteristics of advanced, reliable, and easy to use. Engineer station, operator station, the network communication system, distributed process control system, and historical data recording station are the main components of EDPF-NT+ of distributed control system. The real time system for redundant Ethernet backbone network of double main ring can be fault-tolerant and fast. DPU with I/O communication also use of redundant design, rate of $2 \mathrm{Mbit} / \mathrm{s}$.

\section{DESIGN OF SUPPORT SYSTEM}

By the way of incentive simulation - STS (Support System ), which connects from distributed control system to the model with I/O interface. Almost all the functions are retained over distributed control system. Thus can easily control the research of algorithm analysis. It will be convenient for the model builder of simulator.

The modules set up by support system with the method of independent operation. Data interchange by the opening of shared memory to ensure the rate of data exchange. Data-driven is provided to virtual DPU by support system at the same time. This not only realizes the normal operation of the unit [3], but it make simulating fault and maintaining the current state come true. Thus achieving the effects of back-off operation and repeating. So the actual production process can be better simulated. 


\section{DESIGN OF MULTI-MODEL SYSTEM}

\section{A. The Introduce of STSManageCenter System}

STSManageCenter (Support System Manage Center) system has realized the running of multi-model, and the technical requirements of different models at the same time. Interface of STSManageCenter, as shown in Fig. 1.

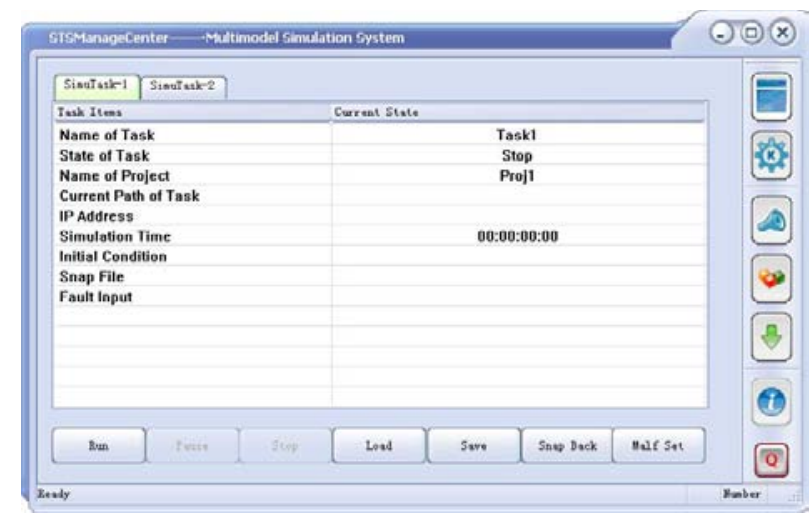

Figure 1. Interface of STSManageCenter.

According to practical requirement, STSManageCenter can start two models at the same time, which can be switched by the tabs of SimuTask. In the items of task, including the task names of simulation, the task status, name of the selected project, the basic information of the path of the current task. The current network address can be configured according to network requirements at the same time.

When STSManageCenter runs, the simulation time can be recorded. During the training hours, the conditions at any time that were saved before can be called through the initial conditions drop-down list items. Snap files are the auto-saved conditions and the files generated that follow the predefined interval. After the model turned off, temporary files will be deleted automatically, and the way avoiding the problem of files growing so large in the long run. The state can be shown when predefined faults launched. Once the fault-files made up, the scene of the accidents can be simulated when training [4].

\section{B. Memory and Computation}

After the basic information of multi-model configured, two pieces of shared memory would be created at the start of the operation, which can be distinguished respectively by different names of models. Meanwhile, two operation thread would be generated, and the corresponding algorithm of the model for real-time operation. The creating processes of shared memory and thread based on computation are shown in Fig. 2.

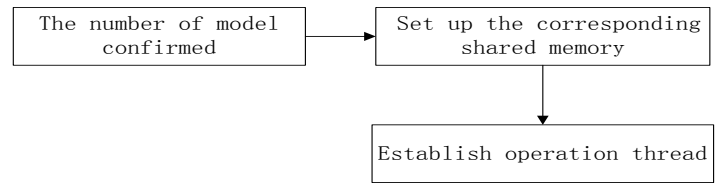

Figure 2. The creating processes of shared memory and thread based on computation.

Shared memory based on multiprocessor computer system [5], the large capacity of memory can be accessed by different central processing unit (CPU). Due to multiple CPU need to access memory quickly, the memory cache will be necessary. Other processors will access to data at any time. Once the cache is updated, shared memory needs to be updated immediately. Otherwise different processors may use different data. And asynchronous data would be generated. It is very convenient to control the data in shared memory. What's more data read-write process is transparent. As a piece of shared memory imported, the current process of user can access in the data with only a string pointer needed to point to.

After the model started up, five pieces of shared memory would be separately set up. Respectively denote AV and DV information, data values, the operation of the memory modules. The structure of shared memory is as shown in table 1 .

TABLE I. THE STRUCTURE OF SHARED MEMORY

\begin{tabular}{|l|l|}
\hline \multicolumn{1}{|c|}{ Properties of Shared Memory } & \multicolumn{1}{|c|}{ Memory Pointers } \\
\hline static information of AV & HFileMapAVMessage \\
\hline static information of DV & HFileMapDVMessage \\
\hline data points of AV & HFileMapAVValuePT \\
\hline data points of DV & HFileMapDVValuePT \\
\hline module's situation of operation & HFileMapOutData \\
\hline
\end{tabular}

The number of multiple models is predefined by the user. Corresponding shared memory can be established after simulation mode simulation support system running. Multi-model communication system (DataCom) can access the data.

\section{Communication of Multi-model}

After each model is set up and running, the corresponding model will launch a program for communication. And the way of point to point communication will be used for DCS of Guodian Zhishen. Point table, generated by the station of EDPF-NT+ engineer of distributed control system. Only model-side points of variables are required to be filled. Relations between data can be generated by the communication system. Then data can be sent in a broadcast way. The communication structure is shown in Fig. 3. 


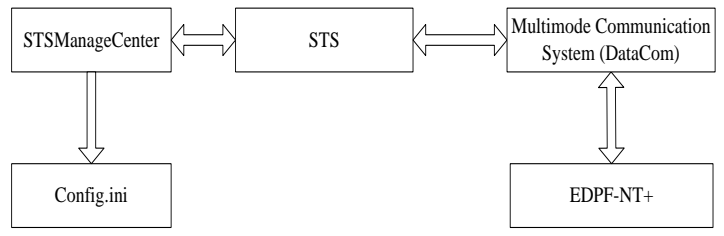

Figure 3. Communication structure.

EDPF-NT+ distributed control system under the way of multiple domain communication, at the time STSManageCenter start up, the configuration information would be read from the file of Config.ini. Data communication thread can be established according to each domain IP by DataCom, the multi-model communication system, so as to realize data parsing individually. Thus the invalid data flow reduced, and the communication efficiency improved.

/**************StartMulti-model $* * * * * * * * * * * * * /$

The following configuration for whether to open multimode function:

/**************StartMulti-model $* * * * * * * * * * * * * /$

[MULTIMODEL]

START $=0$;

/************** SIMUTASK**************/

The following configuration for basic information of each task:

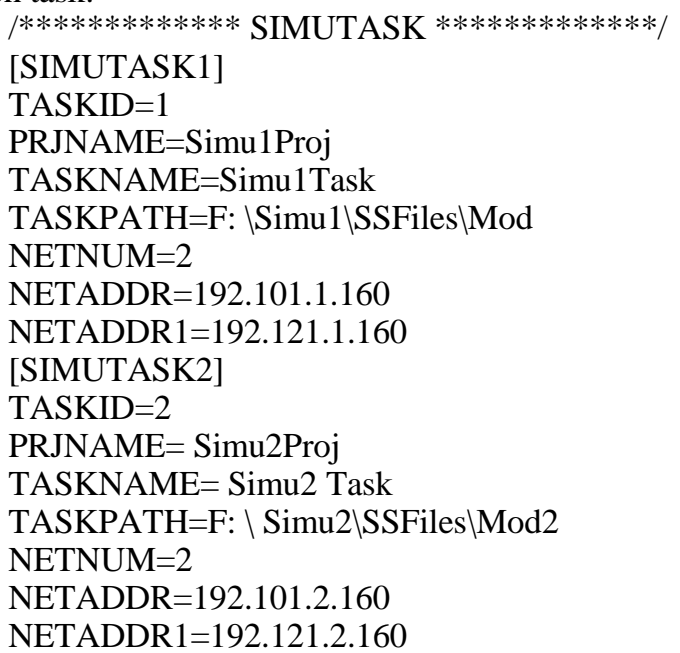

There is no need interfaces between DCS and simulation support system, DPU interacts data directly by multi-model communication system with support system [6]. Each DPU from EDPF-NT+ receive and send I/O data of hardware only in the configuration of its own site. Thus reducing the flow of invalid data to the DPU, the load of DPU network and $\mathrm{CPU}$ can be reduced then. Communication system organizes data according to the station. Each packet contains data only from a station, and by the way of single broadcast station sending to the target. After receiving data, virtual DPU analyzes the ID of each point, and discards the unrecognized data. So even if a packet of data with multiple DPU, DPU can still process normally.
The ports of packets sending side (especially data receiver about target ports) are defined as shown in table 2:

TABLE II. THE PORTS OF PACKETS SENDING SIDE.

\begin{tabular}{|l|l|l|c|}
\hline \multicolumn{1}{|c|}{ Source } & \multicolumn{1}{c|}{ Destination } & \multicolumn{1}{c|}{ Packet Type } & Port \\
\hline Emulation Server & DCS Station & Real Time Data & 6600 \\
\hline DCS Station & Emulation Server & Real Time Data & 6900 \\
\hline Emulation Server & DCS Station & Command & 6900 \\
\hline DCS Station & Emulation Server & $\begin{array}{l}\text { Response Of } \\
\text { Command }\end{array}$ & 6910 \\
\hline
\end{tabular}

Various operating commands can be directly sent from STSManageCenter to DCS, such as the commands of running, pause, stop, and so on. Usually, orders are needed to send to all DPU at the same time. It's okay to send the orders by the way of broadcast because of its small amount. The transfer process of command is shown in Fig. 4.

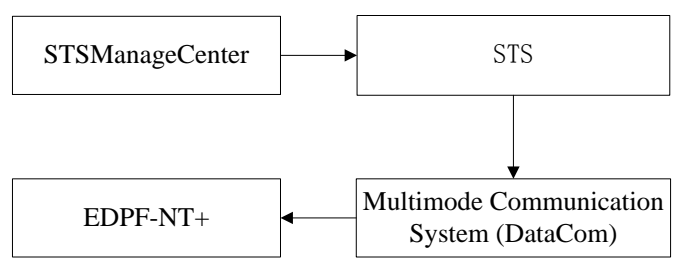

Figure 4. The transfer process of command.

The format of command packet is similar to real time data. It's constructed by the head of 16 bytes and variable data area. The definition of the head is similar to real time data packets. Data area (Commands sent by simulation platform to the DCS) is defined as table 3.

TABLE III. DATA AREA.

\begin{tabular}{|l|l|l|}
\hline Command & Parameters One & Parameters Two \\
\hline int & unsigned int & long \\
\hline
\end{tabular}

V. IMPLEMENTATION OF MULTI-MODEL SYSTEM

Take a 350MW unit, for example. The paper expounds the realization of the function of multi-model. The hardware of power plant multi-model simulation system based on the mode of client/server, communication with $100 \mathrm{M}$ Ethernet-based high-speed network [7]. And realized A and $B$ network redundancy configuration. Model machine adopts a rack-mounted server. The model is DLL PowerEdge R410, with two engineers and eight operator stations. Many sets of DPU to form a cabinet unified, router and power voltage regulator separately stored in a rack. Multi-model simulation system is as shown in Fig. 5. 


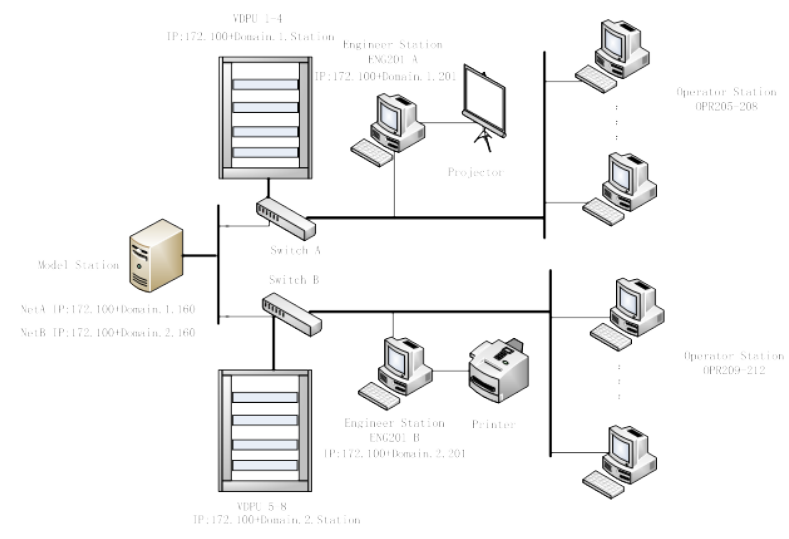

Figure 5. Multi-model simulation system.

Power supply cabinet is generally as part of EDPF-NT+ system, which in charge of the whole power distribution, controlling and protection of EDPF-NT+ system. Power supply to DCS shall be from two different sources. Supplying to the power cabinet firstly and working through the air switch. The power supplies to the control cabinet and work station should be switched by redundant power supplies rapid switcher. But the power sent to the control cabinet is generally not be switched, and supplies by the double-source supply directly.

This instance can realize the dual models running by two groups at the same time [8]. The controlling switches of power cabinet should be started firstly. And the following of model station, engineers and operators starts respectively. The coach only needs to control the station of model. Then the command of the simulation system can be realized. What's more modules can also be changed on line according to the demand. No matter increasing or decreasing. With the fault file set up before, it is easier to have the effect of breakdown according to the mission. Different states of conditions can also be invoked according to teaching demand. And also satisfy the special practice of different states.

\section{CONCLUSION}

The simulation system by adopting STSManageCenter integration simulation support system, with the advanced virtual DPU technology of Guodian Zhishen, the function of multi-model is realized on power plant. STSManageCenter makes the whole process and all operating conditions of real-time simulation come true. It's really a breakthrough on the technology side. The implementation of this system, which greatly reduces the work intensity of the coaches, saves the training time. At the same time EDPF-NT+ system, which is easy to maintain, and running steadily. Considering all about this, the system is worth of widely promoted and widespread use. And in a 350MW thermal power unit simulation, the implementation of multi-model system works well.

\section{ACKNOWLEDGMENT}

I would like to extend my sincere gratitude to my tutors Dong Ze and Wang Dongfeng, for their instructive advice and useful suggestions on my thesis. Special thanks should go to my friends who have put considerable time and effort into their comments on the draft. Last my thanks would go to my beloved family for their loving considerations and great confidence in me all through these years.

\section{REFERENCES}

[1] N. Forouzandehmehr, Z. Han and R. Zheng, "Stochastic Dynamic Game between Hydropower Plant and Thermal Power Plant in Smart Grid Networks," in IEEE Systems Journal, vol. 10, no. 1, pp. 88-96, March 2016.

[2] H. Fang, L. Chen, N. Dlakavu and Z. Shen, "Basic Modeling and Simulation Tool for Analysis of Hydraulic Transients in Hydroelectric Power Plants," in IEEE Transactions on Energy Conversion, vol. 23, no. 3, pp. 834-841, Sept. 2008.

[3] M. S. Donne, A. W. Pike and R. Savry, "Application of modern methods in power plant simulation and control," in Computing \& Control Engineering Journal, vol. 12, no. 2, pp. 75-84, April 2001.

[4] M. A. C. Aghina et al., "Virtual Control Desks for Nuclear Power Plant Simulation: Improving Operator Training," in IEEE Computer Graphics and Applications, vol. 28, no. 4, pp. 6-9, July-Aug. 2008.

[5] F. Casella, "Modeling, Simulation, control, and optimization of a geothermal power plant," in IEEE Transactions on Energy Conversion, vol. 19, no. 1, pp. 170-178, March 2004.

[6] K. Kunitomi et al., "Modeling combined-cycle power plant for simulation of frequency excursions," in IEEE Transactions on Power Systems, vol. 18, no. 2, pp. 724-729, May 2003.

[7] M. S. Donne, A. W. Pike and R. Savry, "Application of modern methods in power plant simulation and control," in Computing \& Control Engineering Journal, vol. 12, no. 2, pp. 75-84, April 2001.

[8] F. Casella, "Modeling, Simulation, control, and optimization of a geothermal power plant," in IEEE Transactions on Energy Conversion, vol. 19, no. 1, pp. 170-178, March 2004. 